\title{
INDICATORS OF INDIVIDUAL AND COMMUNITY MENTAL HEALTH IN WOMEN-HEADED HOUSEHOLDS IN SITUATIONS OF FORCED DISPLACEMENT IN THE DEPARTMENT OF QUINDIOO
}

\author{
INDICATORS OF INDIVIDUAL AND COMMUNITY MENTAL HEALTH IN \\ WOMEN-HEADED HOUSEHOLDS IN SITUATIONS OF FORCED \\ DISPLACEMENT IN THE STATE OF QUINDÍO
}

Recibido: Mayo de 2011 - Revisado: febrero de 2012 - Aceptado: 30 de Mayo de 2012

Por: José Alonso Andrade Salazar ${ }^{1}$, Maira Andrea Sicachá ${ }^{2}$

\begin{abstract}
RESUMEN
Esta investigación tiene como objetivo, analizar el impacto psicosocial que el conflicto armado tiene en la salud mental individual y comunitaria de 30 mujeres cabeza de hogar en situación de desplazamiento forzado en el departamento del Quindío. Para ello se aplicó una ficha de caracterización, la cual indicó que el 53\% de las mujeres desplazadas no siente que controla su vida emocional, un $90 \%$ no accede a redes de apoyo social y el $70 \%$ no cuenta con relaciones de comunicación positiva en la familia. Igualmente, muchas de ellas no tienen oportunidades educativas permanentes $(70 \%)$ considerándose excluidas y señaladas por la comunidad $(47 \%)$. El estudio indicó que el $80 \%$ no ha solicitado asistencia psicológica y que un $67 \%$ piensa que la comunidad favorece el acceso a recursos y servicios por lo que su vida cultural y social es activa $(70 \%)$.
\end{abstract}

\section{PALABRAS CLAVE:}

Conflicto armado, Desplazamiento Forzado, Salud Mental Individual, Salud Mental Comunitaria, Mujeres Cabeza de Hogar.

\section{ABSTRACT:}

This research aims to analyze the psychosocial impact caused by the armed conflict both on the individual and the community mental health of 30 womenheaded households in a situation of displacement in the State of Quindio. For such a purpose, a characterization card was applied, which showed that $53 \%$ of displaced women are not able to control their emotional life, $90 \%$ do not have access to social support networks, and $70 \%$ do not have positive communication relations within the family. Also, many of them do not have permanent educational opportunities (70\%) considered themselves excluded and pointed at by the Community (47\%). The study indicated that $80 \%$ did not request psychological assistance and $67 \%$ think that the community favors the access to resources and services, which makes their cultural and social life active $(70 \%)$.

\section{KEY WORDS:}

Armed Conflict, Forced Displacement, Individual Mental Health, Community Mental Health, Women-Headed Households.

\footnotetext{
${ }^{1}$ Psicólogo. Especialista en Gestión de proyectos de desarrollo. Docente investigador. Coordinador de investigaciones del programa de psicología de la Universidad De San Buenaventura extensión Ibagué - Convenio Universidad San Martín. Colombia 2012. Email: 911 psicologia@gmail.com - Investigador del grupo PSODELICA (GrupLAC) en la línea de investigación Psicología y sociedad.

${ }^{2}$ Estudiante investigadora de décimo semestre del programa de psicología de la Universidad de San Buenaventura Colombia extensión Armenia en convenio Universidad San Martín. Investigador del grupo PSODELICA (GrupLAC) en la línea de investigación Psicología y sociedad. Email: andru-90@hotmail.com
} 


\section{Introducción}

El conflicto armado en Colombia ha generado cambios sociales y culturales que afectan principalmente la salud mental de quienes se han visto impactados por la dinámica terrorista de la violencia. Estas poblaciones a menudo son comunidades históricamente excluidas de los beneficios socioeconómicos de la sociedad de producción y consumo, es decir, campesinos, agregados de fincas, pequeños agricultores, mineros, comunidades indígenas y afrodescendientes, parceleros y personas pobres de los cascos urbanos que no simpatizan con la ideología y el estado de sitio que impone el grupo armado dominante. Los lugares de origen de las familias desplazadas son zonas rurales cuya productividad "agricultura, minería, animales y comercio portuario", capital humano "padres, madres, niñas y niños como posibles soldados", ubicación geológica "zonas de paso estratégico de tropas" y presencia de minerales, se constituyen en objetivos económicos para los grupos armados ilegales, lo cual los vuelve objetivos militares de los actores sociales del conflicto.

El desplazamiento es un fenómeno devastador que se asocia a la historia de violencia, inequidad de género y a la violación a los derechos humanos en Colombia, "estimativos indican que más de la mitad de los 5 millones de desplazados en el país son mujeres y de ellas, 4 de cada 10, han sido desplazadas por el conflicto armado" (Córdoba \& Londoño, 2005, p.4). Según Acción Social, entre 1997 y 2009, en Colombia se han registrado 3.303.236 personas en situación de desplazamiento. De esta cifra, 1.623.236 son niñas o mujeres adultas y de este total 1.183 .908 tenian menos de 18 años cuando fueron obligadas a desplazarse (36\%) (REDANDI. 2010). Las múltiples guerras internas han llevado a la población colombiana a movilizaciones colectivas que buscan proteger la integridad fisica, la seguridad familiar y la reintegración de sus derechos ya que, las personas desplazadas se ven directamente amenazadas por grupos armados insurgentes especialmente las mujeres y niños. Es importante mencionar que, de acuerdo con Unicef (2007) en Colombia, de cada 100 personas desplazadas, 70 son mujeres junto a sus hijos e hijas y más del $50 \%$ de la población desplazada durante los últimos seis años son niñas y niños, de los cuales, cerca de 10 de cada 100 son víctimas de minas antipersonales.

La realidad de las madres cabeza de hogar es poco visibilizada en todas las regiones del país, condición que se deriva de los imaginarios sociales respecto a los roles femeninos y la estructura patriarcal con la que opera el conflicto. Según Acción Social en el departamento del Quindío en el año 2010 hubo 1102 personas y 362 hogares en condición de desplazamiento que llegaron a diferentes municipios a pesar de que estos no permiten la satisfacción completa de las necesidades básicas. La escogencia de estos lugares eleva los indices de vulnerabilidad y pobreza por efecto del desempleo, maltrato, desnutrición y la desestructuración familiar. Las movilizaciones masivas hacia el Quindio provocan un desequilibrio demográfico en las ciudades receptoras, factor que ha posibilitado el incremento en los índices de vulnerabilidad a razón de las dificultades que emergen de la convivencia comunitaria y las desigualdades económicas, educativas y culturales entre grupos, factores que se suman a notables dificultades de integración a nuevos ambientes y el asentamiento familiar en zonas hostiles y deprimidas tales como, laderas urbanas, ollas citadinas e invasiones.

El desplazamiento forzado produce un cambio extremo en el sistema de creencias, representaciones y en la vida psicológica de las víctimas, causando una reestructuración importante de la psique y las dinámicas familiares, especialmente en las madres cabeza de 
hogar. Para Profamilia (2010) una característica de los hogares desplazados es la feminización de la jefatura, pues en 1995 , una cuarta parte $(24 \%)$ de los jefes de hogar eran mujeres, en el 2000 el 28\%, en el 2005 del 30\% y en el 2010 fue del 34\%. Los resultados confirman la tendencia creciente en la jefatura femenina de los hogares colombianos, tanto en zona urbana como rural. Igualmente, la II Encuesta Nacional de Verificación (II-ENV) señaló que el desplazamiento tiene un proceso de "feminización" ya que el 42,2\% del total de hogares desplazados tiene jefatura femenina, de estos, el 78,8\% es jefatura única, es decir, mujeres viudas, separadas o abandonadas en el proceso del desplazamiento forzado (CODHES. 2008, p.3).

A menudo para las familias desplazadas por la violencia del conflicto armado, la única salida resulta en "cruzar las fronteras, despojarse de sus tierras, y abandonar su memoria histórica para sobrevivir, salvando a los suyos de una realidad ineludible y constante" (Álvarez \& Cols, 1999, p. 47); sin embargo, cuando logran escapar no solo son perseguidos por los grupos armados sino también por el peso del estigma que implica la condición de desplazamiento, por lo que muchas familias quedan expuestas en su migración a la discriminación y exclusión social de las comunidades receptoras. Se puede constatar que "tras la huida, el desplazado tiene que enfrentar la vida fuera de su ambiente, sin recursos de ningún tipo a veces hacinado en asentamientos donde el alimento recibido, termina generando dependencia, y la falta de un trabajo autónomo puede desembocar en abulia y pasividad" (Baro. I. 1984, p. 5)

\section{Metodologia.}

Esta investigación es de corte empírico - analítico, de tipo descriptiva, con un diseño descriptivo - transversal, el cual pretende identificar y describir las consecuencias individuales y colectivas a la salud mental, derivadas del desplazamiento forzado, para lo cual se tipificaron los indicadores en salud mental individual y comunitaria tomando en cuenta su prevalencia en las mujeres cabeza de hogar, victimas de desplazamiento forzado en el departamento del Quindío en el año 2010. La investigación se realiza en un momento único en el tiempo, llegando a medir las variables a partir de la experiencia individual de las mujeres evaluadas, deduciendo las relaciones entre las variables estudiadas, tal y como se dan en su contexto natural.

\section{Participantes.}

La muestra estuvo compuesta por 30 mujeres en situación de desplazamiento forzado que asisten a las oficinas de la unidad de atención y orientación al desplazado (UAO) seccional Quindío ubicada en el municipio de Armenia. Las personas fueron escogidas a través de muestreo aleatorio por conglomerados en donde la población está integrada en grupos específicos, determinados por ser madres cabeza de hogar, con uno o más desplazamientos de tipo individual y masivo, provenientes de diferentes partes del país, mayores de edad y de diversas etnias (Indigena, Afrodescendiente, Raizal y Mestiza).

\section{Instrumentos.}

El instrumento aplicado, para la recolección de datos sociodemográficos fue una ficha de caracterización que tuvo en cuenta variables como: edad, género, etapa del ciclo vital, tipo de desplazamiento, motivos del desplazamiento y empleo. Igualmente se usó una segunda ficha con indicadores de salud mental individual y comunitaria, que contaba con ítems como 
seguridad económica y social, manejo de las relaciones en el hogar, control emocional, redes de apoyo, participación comunitaria, vida cultural y social, entre otros. La prevalencia de estos indicadores, dio cuenta del estado actual de la familia desplazada respecto a su salud mental "individual y comunitaria" a partir de la percepción de la madre cabeza de hogar. La información fue tabulada y analizada a través del programa Excel de Microsoft, tomando en cuenta los porcentajes prevalentes y su correlación estadística.

\section{Procedimiento.}

El instrumento fue entregado a las madres cabeza de familia desplazadas de forma individual. Se les expresó que la ficha era diligenciada de manera anónima y que disponían del tiempo máximo de media hora para responderla. También se resolvieron posibles dudas respecto a su contenido, explicando lo que evaluaba cada Ítem. Se entregó a cada persona un consentimiento informado y tras la firma se procedió a aplicar la ficha. La tabulación y análisis de datos se hizo a través del programa Excel de Microsoft Windows (datos agrupados, gráficos de frecuencia etc.)

\section{Resultados.}

El desplazamiento forzado tiene consecuencias graves en la calidad de vida de las mujeres víctimas del conflicto armado. Entre las secuelas notorias se encuentran: desempleo (83\%), inseguridad financiera y laboral (80\%) y una inadecuada comunicación intrafamiliar $(70 \%)$. De acuerdo a los resultados de la ficha de caracterización el $13 \%$ de las mujeres son indígenas, el 7\% Afrodescendientes, un 3\% raizal (se entienden aquellos como aquellos habitantes que tienen una identidad definida basada en su historia, sus manifestaciones culturales, su lengua (el sanandresano) y su identidad) y el 53\% mestiza. En esta población existe desplazamiento masivo (20\%) aunque la movilización predominante es de tipo individual (80\%), por motivos como el hostigamiento familiar $(73 \%)$, escape por encajonamiento $(10 \%)$, acoso sexual en mujeres y niños $(10 \%)$, asesinato de un familiar (17\%), persecución socioeconómica (13\%) y tierras como objetivo militar (13\%).

Respecto a los Indicadores de Salud Mental Individual, se encontró que el 53\% de las mujeres encuestadas "no se sienten dueñas de sí mismas, ni controladoras de su entorno", igualmente un $90 \%$ no tiene la posibilidad de acceder a redes sociales de apoyo, factor que se relaciona con el hecho de considerar que la comunidad no favorece el acceso a recursos y servicios (33\%); el estudio indica que el $80 \%$ de las mujeres no tiene la posibilidad de acceder a servicios básicos, ni cuentan con asistencia en salud mental (80\%). En cuanto indicadores de salud mental comunitaria se encontró que el $60 \%$ tiene un ambiente físico amable, confortable y acogedor que favorece el crecimiento sano de sus hijos y familia (73\%), presentando una vida cultural y social activa (70\%). De ellas el $67 \%$ evita participar de la democracia local al tiempo que, un $47 \%$ considera que su entorno comunitario presenta exclusión, señalamiento y/o desconfianza con la familia desplazada.

En relación al soporte obtenido de las instituciones, se encuentra que el $77 \%$ ha recibido apoyo económico de acuerdo a su condición de Madre Cabeza de Hogar (MCH) desplazada, por lo que consideran que sus demandas y necesidades asistenciales han sido atendidas con eficacia por las instituciones $(77 \%)$. La atención en salud está referida al plano orgánico en el $100 \%$ de los casos, por lo que el factor psicológico es tomado en cuenta sólo cuando se sobreañade a una condición física patológica (20\%), en cuyo caso se asume como 
consecuencia y no como causa; por consiguiente, el $80 \%$ de las mujeres en situación de desplazamiento forzado, nunca ha solicitado atención con un profesional en salud mental por problemas psicológicos, consejería o asesoría para ellas o para su familia.

\section{Discusión.}

Las $\mathrm{MCH}$ que conforma la población estudio de esta investigación, escaparón del hostigamiento familiar y se movilizaron de sus tierras hacia el departamento del Quindío en busca de nuevas y mejores oportunidades, protegiendo sus familias y su identidad, demostrando un gran ingenio y resistencia a la hora de afrontar las adversidades y de luchar por la supervivencia de sus familias en circunstancias hostiles y desconocidas. Las acciones terroristas tienen un efecto devastador en la formación constante de la integridad de la mujer, limitando las oportunidades de estas para el desarrollo personal y el cumplimiento de sus proyectos de vida. "Los traumas se asocian a la violencia que precede generalmente los desplazamientos, el cambio precipitado en la administración de su tiempo y espacio, y el desmembramiento del núcleo familiar genera una nueva situación aún más hostil" (Ballesteros \& Cols, 2006. p. 8).

De acuerdo a Aguilera \& Torrado (2001) el conflicto armado se transforma en el enemigo acérrimo de la memoria, el patrimonio cultural y la identidad de los pueblos, ya que crea nuevas formas particulares de ver y sentir la ciudad, de añorar el campo, además de generar miedos desconocidos tales como: el miedo al otro, a su forma de ver el mundo, un miedo al ruido de la ciudad y hasta al amanecer, elementos que traen consigo la incertidumbre de la supervivencia fisica y la desconfianza ante el entorno. El choque emocional y social lleva a que la vida del desplazado esté marcada por la angustia, el miedo y el dolor, motivos que traen consigo consecuencias persistentes y dejan heridas que se atenúan a lo largo del tiempo pero que pueden convertirse de manera gradual en secuelas imborrables para quien las vive. Las minorías étnicas siguen presentando más obstáculos para vincularse a trabajos formales porque enfrentan una doble discriminación: "por su condición de desplazados y por pertenecer a una minoría étnica" (Ibáñez \& Velásquez, 2008, p.25), así, el desempleo post desplazamiento afecta la satisfacción de las necesidades básicas y aumenta el nivel de tensión de la familia, lo cual se ve reflejado en una creciente inseguridad afectiva, financiera y laboral, pues muchas de las habilidades de la población desplazada no son demandadas en el mercado laboral urbano, lo cual retrasa la inserción laboral de esta población; "en los casos en los que logran vincularse es por lo general, en el sector informal que no ofrece estabilidad laboral, son mal remunerados y no tienen prestaciones sociales" (p.20).

Las mujeres que encuentran una ocupación temporal o permanente lo hacen en el servicio domestico, el comercio a baja escala, el sector informal y en algunos casos cuidando menores y ancianos; es importante mencionar que la mayoria de mujeres logran conseguir un trabajo antes que los hombres pues "los parámetros tradicionales que dejaban en manos de los hombres el sostenimiento del hogar, han cambiado, convirtiendo a la mujer en la nueva proveedora de recursos" (Álvarez \& Cols, 1999, p.83). El rol tradicional del hombre como proveedor absoluto y de la mujer dedicada sólo a las actividades domesticas ha cambiado debido a que muchas de las mujeres en situación de desplazamiento son viudas, separadas, abandonadas o madres solteras, antes o durante el desplazamiento, lo cual es determinante en el incremento de hogares con jefatura femenina a razón de la muerte, desaparición o desplazamiento de la pareja. Los roles se invierten porque el conflicto armado propicia una resignificación de la patriarcalidad en el contexto intrafamiliar, de acuerdo con Guevara \& 
Barney (2009) los roles de comportamiento tradicionales han variado porque la mujer actual, en esa adaptación a lo urbano ha desarrollado aptitudes y habilidades propias que, por mucho tiempo fueron ocultadas y "que en este nuevo espacio surgieron como un medio para obtener ingresos económicos o conseguir apoyo para sus procesos de emprendimiento" (p.64).

Esta "inversión de roles" puede resultar contraproducente sin un proceso previo que sostenga el cambio ideológico de la sociedad, acerca de actividades estereotipadas, femeninas y masculinas (Gandulfo. 2011), puesto que, a menudo, el cambio trae consigo un profundo rechazo de la sociedad y las comunidades desplazadas con esquemas de relación tradicional de tipo patriarcal. Al respecto Ignacio Martín Baró (2000) señala que en el análisis del proceso histórico de la construcción de la identidad sexual, se pone en evidencia una diferenciación discriminatoria que, en sociedades como la latinoamericana niega la alteridad real de la mujer, subordinando su desarrollo y proyecto de vida al desarrollo y proyecto vital del hombre, produciendo relaciones de dependencia y opresión. Por tanto, su deconstrucción se realiza sobre la ideología patriarcal dominante, conflicto que puede ir resolviéndose con la construcción de una nueva subjetividad que no está basada en la dicotomía hombre-mujer. Las madres son quienes conservan esta cultura aunque en realidad son portadoras de las dos: "una situación matristica en el seno de la familia y un situación patriarcal en la vida adulta" (Maturana, H. 1991, p.23)

Frecuentemente la función maternante de protección y apoyo se ve desviada por sucesos traumáticos que modifican las dinámicas de relación al interior de las familias, e implican una redistribución de los roles donde las mujeres pasan de ejercer el papel de madres protectoras, para desarrollar roles paternales que implican actitudes de fuerza, constricción ambiental y el sustento diario de la familia (Andrade \& Cols. 2011, p.19); como resultado se tiene que, a menudo la mujer enfoca su energía en la búsqueda de empleo, antes que, en el contacto con miembros de su familia de origen, las relaciones interpersonales (Sullivan. 1954) y/o la búsqueda de una nueva pareja, aislando aún más su vida emocional. Esta situación lleva a que las MCH no se sientan dueñas de sí y ni controladoras de su entorno, lo que indica que quienes han sufrido un proceso de violación de sus derechos modifican la percepción que tienen de sí mismos, porque el trauma del evento sumado a los problemas de los lugares receptores y/o de reasentamiento no brindan los recursos económicos, sociales de apoyo y sostén a la familia aumentando su condición de vulnerabilidad. La familia desplazada, orientada por la $\mathrm{MCH}$, comienza a vivir una situación en la que se confunde el presente con el pasado y en la que hay sincretismos culturales y pérdida de la confianza.

En este sentido, la persona en situación de desplazamiento forzado no logra encontrar un espacio para elaborar y superar sus pérdidas "lo cual lleva a que su pasado se congele, puesto que su nueva situación le imposibilita asumir el presente, construir el futuro y un proyecto de vida individual y social" (Serna \& Ramírez. 2007, p.10). El impacto del desplazamiento sobre la identidad, autonomía y dignidad coloca a las $\mathrm{MCH}$ en condiciones de mayor vulnerabilidad respecto al resto de la población, ya que "las mujeres y los hombres deben abandonar no solo el territorio y su historia personal, sino escapar sin nombre para huir de la persecución y el peligro de ser identificados" (Córdoba \& Mejía 2005, p.4). De acuerdo con Médicos Sin Fronteras (2010) las $\mathrm{MCH}$ deben convivir con una comunicación inadecuada, relaciones conflictivas y/o desestructuración grave (separación traumática, cambios bruscos en la dinámica familiar, etc.). Además de que los espacios de privacidad familiar se reducen y las relaciones no se fortalecen, manteniendo flotante el dolor que todos sienten y que ninguno comunica, factor potenciado por el hacinamiento y la falta de privacidad de los espacios 
adecuados para el descanso, pues "la convivencia en espacios altamente hacinados reduce ostensiblemente las relaciones intra-familiares de intimidad y privacidad, se acrecientan las tensiones y angustias, y en muchas ocasiones los problemas intrafamiliares tiene que ventilarse en público" (Guevara, 2003, p.11)

El hacinamiento representa incomodidad, pérdida de privacidad, conflicto por el uso del espacio y en muchos casos convertir a los otros semejantes en los otros próximos (Castillejo. 2000) lo que genera conflictos y deteriora las relaciones humanas. Para sobrevivir en la ciudad muchas familias se ven obligadas a vivir con familiares o vecinos, lo cual crea "continuos enfrentamientos por los estilos de crianza, el ejercicio del control y el castigo" (Castillejo. 2000. Citado por Bello. M. 2004, p.3). La mujer, como jefe cabeza de hogar, además de asumir su rol de madre, hija o hermana debe hacerse cargo de responsabilidades económicas y familiares, entre las que se encuentran la búsqueda de alimentos, el contacto con personas, comunidades e instituciones y la adquisición de vivienda. Concretar un espacio para vivir es una de las tareas más complejas debido a los costos exagerados en el área urbana, lo cual instaura una angustia permanente por el poco presupuesto familiar y el aumento de la pobreza, en consecuencia, las familias tienen como última alternativa el arriendo en condiciones de hacinamiento en áreas peligrosas, generando un nivel menor de bienestar y un mayor riesgo para la salud de todo el núcleo familiar.

La familia tiene a cargo la reproducción social y afectiva del lenguaje, por ello la $\mathrm{MCH}$, a partir de su rol, genera apoyos inter comunitarios e interacciones psicosociales que proporcionan elementos de ajuste familiar y control social para obtención de bienes y servicios, esta actividad es positiva "haciendo que se mantenga una actitud de apoyo, facilitando que en la familia se elaboren mecanismos para afrontar la situación de desplazamiento, como la unidad y el apoyo mutuo como función de protección" (Viveros. E. 2010, pp.9-13). De acuerdo con el estudio, el desplazamiento propicia en las jefes de hogar la idea fatalista respecto a la continuidad de las necesidades y las violaciones a sus derechos; al tiempo que, las motiva ante la idea de reestructurar su existencia, si bien la mujer desplazada atraviesa momentos confusos en los que no logra ajustarse a su nueva realidad, sus habilidades para obtener ayuda, trabajo y criar los hijos son mayores que muchos hombres, que rápidamente escapan ante la saturación de responsabilidades del hogar.

Las poblaciones desplazadas están a menudo confinadas a lugares de elevada peligrosidad que aumentan los factores de riesgo para sus familias, derivados de la pobreza y la multiplicidad de problemas urbanos. Las $\mathrm{MCH}$ encuentran dificultad para formar redes de apoyo, factor que proviene "tanto de la exclusión y marginación que viven los grupos étnicos del país, como de las estructuras socioeconómicas discriminatorias y racistas prevalecientes, y de la desintegración de sus redes sociales, comunitarias y culturales" (Cepeda. 2008, p.318); en consecuencia, la función de las redes de apoyo dirigida a mejorar la capacidad de resiliencia individual y comunitaria, constituir contactos, manejar el rumor negativo, elaborar propuestas, pedir ayuda y exigir la reivindicación de sus derechos, se disocia en aspectos imaginarios como la idea de un estado que ayuda, pero desprotege y de personas que los aceptan pero los excluyen. Correlativamente la naturaleza y conformación de las redes sociales en lo rural y urbano están signadas por diferencias entre los escenarios de encuentro, metalenguajes, aspectos histórico-culturales y emociones propias de la emergencia de estigmas, prejuicios o resistencias para integrarse a los grupos. 
De acuerdo con los hallazgos en lo rural las relaciones comunitarias incluyen a todo el grupo familiar, están lideradas por los roles de hombres y mujeres, padres y madres de familia y son definidas dentro de los espacios dedicados a las actividades de recreación y religiosidad. Es decir que, en las condiciones actuales el campesino desplazado no logra pertenencia social en el barrio urbano porque el tejido social, con el que cuentan, es frágil debido al desarraigo forzado y las diferencias emergentes entre la naturaleza de la interacción social "referente" y la interacción social existente, "consecuentemente, las redes de apoyo de tipo comunitario propias del campo son reemplazadas por las instituciones y organizaciones humanitarias" (Riaño, 2006. p, 137-138). Las MCH desplazadas buscan en las redes sociales la integración a grupos que operen como unidad colectiva, a través de la expresión de actitudes, afectos y comportamientos comunes, pues en ellas se describe lo cotidiano del vínculo social a través del apoyo y el sostén comunitario. En éste sentido el carácter de permanencia del vinculo social propicia la convivencia social y el acuerdo intra e intercomunitario.

La investigación encontró que los indicadores comunitarios de salud mental, son percibidos de manera positiva debido a que las $\mathrm{MCH}$ cuentan con el apoyo de otras familias en su misma condición de desplazamiento como estrategia para prevenir el deterioro del tejido social y favorecer su fortalecimiento, por lo cual consideran que la comunidad beneficia el acceso a recursos y servicios, lo que indica que su vida social y cultural trata de ajustarse, condición que da cuenta de sus habilidades de comunicación y resiliencia. En estos escenarios de encuentro se crean nuevas relaciones interpersonales, porque las personas tratan de encontrar la mejor manera de canalizar sus represiones y superar sus duelos. Las MCH víctimas del conflicto colombiano "no sólo sufren las consecuencias de la violencia directa, sino también de la violencia indirecta manifiesta a través del silencio y el estigma comunitario, condición que dificulta el reconocimiento social de la condición de víctima. El conflicto armado genera personas con problemas de sentido de pertenencia e identidad ya que "no hay integración social y por tanto se complica su adaptación al medio y la superación de los eventos traumáticos (...), acentuando las pocas garantías que tienen de restablecimiento social, económico y emocional" (MSF. 2010, pp.16-18).

A diferencia de otras mujeres que llegan con su familia, las $\mathrm{MCH}$ en su mayoría provienen del sector rural, algunas no han tenido acceso a educación permanente y presentan un retraso educativo porque "perdieron el interés" o no sienten que pueden cumplir y/o culminar el ciclo educativo normal. Una cantidad importante de mujeres que vienen de las zonas rurales ni siquiera han terminado el ciclo básico, lo que limita sus oportunidades laborales en ciudades o cabeceras municipales porque las capacidades y oportunidades de empleo están muy relacionadas con el nivel educativo y la adquisición de habilidades específicas o especializadas. En éste sentido Gómez (2009) considera que la educación ofertada a las mujeres desplazadas no tiene en cuenta los horarios del cuidado y organización del hogar, por ello los programas de "alfabetización y educación dirigido a mujeres adultas no tienen ningún impacto, ellas prefieren seguir con el cuidado de la familia y el trabajo al que logran acceder" (Gómez citada por Ocampo. 2009, p.89) en este sentido la educación no es una primera decisión a tomar respecto a su nueva situación.

Respecto a los problemas en Salud Mental se debe tomar en cuenta que el estado psíquico es directamente proporcional a la magnitud del evento y la capacidad individual y comunitaria para hacer frente a la angustia y/o procesar esos contenidos persecutorios que resultan de la condición de vulnerabilidad en aumento de las víctimas, pues "en un principio, el desplazamiento trajo como secuela depresión y ansiedad, trastornos de salud poco comunes, 
los cuales fueron incidiendo en la armonía del grupo y en las relaciones familiares" (Guevara \& Cols, 2000. p.10). Esta incidencia tiene grados de afectación diversos en cada miembro familiar, siendo muy nociva en las $\mathrm{MCH}$ porque "las mujeres sobrevivientes (...), se escabullen de la violencia o resisten hasta el límite de sus posibilidades humanas y a menudo huyen cuando la unicidad mente-cuerpo se disocia y la voluntad se quebranta totalmente" (Andrade, J. 2010, p.44); los problemas de salud mental emergen a la par con la presión de los grupos armados y el sentido anulativo de la violencia, causando en la mujer en situación de desplazamiento forzado una alteración de "la identidad, porque ya no le es posible reconstruirse a través de sus representaciones, roles, costumbres, y legados generacionales" (p. 44) propios de su actividad socio familiar.

Guevara \& Cols (2009) consideran que "las deficientes condiciones de salud afectan a las mujeres y limitan su posibilidad de vinculación a procesos productivos, y al no estar inscritas en el sistema de salud genera unos gastos extras en medicamentos que no se pueden cubrir, lo que las ha obligado a recurrir a su sistema de salud tradicional, en sintesis "a las mujeres se les ha dificultado tener acceso a la salud, a la educación, al trabajo, así como tomar sus propias decisiones; también han perdido identidad, confianza y su vinculación a redes sociales, lo que las hace vulnerables, pues se les dificulta hacer valer sus derechos" (p.64). Es importante anotar que las MCH escasamente acuden a un profesional en salud mental, lo que ha favorecido en ellas la emergencia y consolidación de trastornos de tipo somatomorfo, pues el cuerpo se ha convertido en el espacio simbólico de inscripción del trauma y en el medio de canalización de problemáticas inmanejables; en este sentido el factor psicológico se toma en cuenta cuando se sobreañade a una condición física patológica, en cuyo caso se asume como consecuencia y no como causa, reprimiendo el hecho de que éste se origino bajo condiciones de malestar social propios de la presión del conflicto armado y los actos de lesa humanidad.

No solo las experiencias pasadas dificultan los procesos de adaptación a la comunidad receptora sino también, el hecho de que en su condición de desplazadas las mujeres manifiestan haber sido excluidas, estigmatizadas y señaladas a todo nivel en "espacios donde la persona y/o la familia, no siente legitimidad ni poder para desencadenar la frustración contenida, por lo que la familia se convierte en la canalizadora "de mano" de una frustración insoportable, intensa y descontrolada" (Andrade, J. 2010, p.12) lo cual aumenta la crisis interna por la que pasa en núcleo familiar; este factor se suma a la posición peyorativa de ciertos conglomerados sociales que por falta de empatía social están lejos de entender la irracionalidad del conflicto y la creciente problemática biopsicosocial de las personas en situación de desplazamiento.

\section{Conclusiones y Recomendaciones}

El conflicto armado tiene graves consecuencias que afectan especialmente a la mujer, entre las que se encuentran el deterioro de la comunicación familiar positiva, la perdida de privacidad por hacinamiento, los trastornos psicológicos y enfermedades orgánicas que emergen o se cronifican por efecto del estrés, la búsqueda en los hijos de patrones de identificación externos que pueden resultar disfuncionales y el aumento de la vulnerabilidad psicosocial a toda escala. En ese sentido, el desempleo post desplazamiento en la mujer es elevado pues, las posibilidades de ocuparse son bajas, factor que afecta la satisfacción de las necesidades básicas y aumenta el nivel de tensión intrafamiliar. El desplazamiento tiene efectos devastadores para la integridad de las $\mathrm{MCH}$ limitando las oportunidades para el desarrollo personal y el cumplimiento de sus proyectos de vida, por tal motivo las mujeres 
desplazadas continúan ejerciendo un rol tradicional de cuidado y acompañamiento pero lo modifican sustancialmente asumiendo de manera transitoria o permanente, múltiples responsabilidades que responden a la necesidad urgente de sobrevivir y sostener emocional y materialmente a sus familias.

En las MCH la salud mental individual es frágil pues, la continuidad de los sucesos de vulneración de sus derechos en los nuevos escenarios de asentamiento y las secuelas dejadas por la violencia antes, durante y después del desplazamiento, son determinantes para la estructuración de cuadro clínicos psicopatológicos. A diferencia de lo individual, a nivel de salud mental comunitaria, las madres cuentan con el apoyo de otras familias desplazadas, lo cual previene el deterioro del tejido social y lo fortalece a fin de conservar un principio de realidad ajustado al lenguaje y las dinámicas comunitarias de su entorno. Muchas MCH no experimentan directamente la exclusión, el señalamiento y la desconfianza, lo cual se ve relacionado con el ejercicio de una vida social y cultural activa. De acuerdo a lo expuesto es importante considerar el desplazamiento forzado como una problemática multidimensional (social, económica, cultural, política y de salud orgánica y mental) por lo cual se deben realizar programas de atención-intervención encaminados a contrarrestar el sufrimiento emocional y no solo a "mitigar" descompensaciones durante las crisis emocionales.

Por tal razón, estos programas deben ser integrales a fin de proporcionar una mejor calidad de vida generando espacios de desarrollo laboral y emocional a nivel individual, familiar y comunitario. En lo individual la atención psicosocial debe ofrecer espacios para que las $\mathrm{MCH}$ recuperen su autoestima, realicen adecuadamente sus procesos de duelo por las pérdidas materiales, simbólicas y afectivas, favorecer el restablecimiento de sus proyectos vitales y estimulando la participación activa de los procesos de cambio social y participación ciudadana. Los proyectos productivos son también proyectos afectivos pues, buscan la autorrealización psicoafectiva y material, fomentando la búsqueda de nuevas alternativas para el mejoramiento de su calidad de vida. Las MCH deben tener conocimiento de sí mismas y de su actitud frente a la situación biopsicosocial de sus familias, con lo que se busca fortalecer la capacidad individual y colectiva para confrontar y resolver los conflictos, además de pensar en la construcción de un mejor futuro, buscando soluciones y alternativas de desarrollo que permitan la recuperación emocional y rompan los círculos de dolor y sufrimiento al crear esquemas sanos de relación intrafamiliar y social.

En el ámbito familiar la atención psicosocial debe fortalecer las relaciones intrafamiliares con el propósito de disminuir la desintegración familiar y la aceptación de la nueva realidad psicosocial de la familia, fomentando relaciones afectivas adecuadas y de respeto, que brinden a los miembros una mayor estabilidad emocional a través de la comunicación asertiva y el establecimiento de lazos afectivos familiares y social-comunitarios duraderos. A nivel comunitario, la atención psicosocial debe procurar promover la convivencia a través de la prevención del deterioro del tejido social, detectando oportunamente los conflictos que amenacen la identidad, la comunicación y la perdida de intereses comunes, proceso que debe sustentarse a través del desarrollo comunitario y la convivencia pacífica, lo cual implica brindar estrategias de afrontamiento y el desarrollo de redes de apoyo que actúen eficientemente en situaciones de refuerzo ante los logros, apoyo de iniciativas e intervención en momentos tensión y estrés ambiental.

Uno de los caminos para el logro de una atención efectiva proviene de la recuperación de la 
memoria colectiva (prácticas societales, rituales de transición y tradición oral) mediante la conformación de grupos intereducativos (capacitación de lideresas comunitarias) que multiplican los aprendizajes y conforman con la colectividad redes de apoyo que buscan la reintegración del sentido de pertenencia sociocultural, la solidaridad y la cooperación comunitaria. Es importante proporcionar información veraz y actualizada a la comunidad desplazada y hacer el acople con la población receptora, buscando fortalecer el apoyo local, los factores protectores y las habilidades de resiliencia. Los programas de asistencia deben disminuir el sufrimiento personal y grupal (familiar y comunitario) de las $\mathrm{MCH}$ enfatizando en la identificación e intervención de problemas, necesidades y demandas psicosociales de las víctimas, tomando en cuenta que las posibles soluciones provienen del análisis biopsicosocial de las procesos de interacción entre personas, cultura, grupos y el medio ambiente. 


\section{Referencias Bibliográficas.}

Acción Social. (2010). Reporte general por género. Personas incluidas según género. Fecha de corte: 26/02/2011. Disponible en:

http://www.accionsocial.gov.co/EstadisticasDesplazados/GeneralesPD.aspx

Álvarez Correa, M. (2000). Raíces Sin Tierra. Atención e Impacto del Desplazamiento Forzoso. Procuraduría Delegada para la Defensa del Menor y la Familia.

Amaya Cañola, A \& Rueda, R. (2005). Las mujeres cabeza de hogar victimas del desplazamiento forzado por la violencia. Desplazamiento forzado por la violencia en Colombia contexto. Universidad nacional de Colombia sede Medellín. Descargado de: http: / / agora.unalmed.edu.co/desplazados / canal3/proyectos / 2005 01/individual/Las\%20mujeres\%20cabeza\%20de\%20hogar\%20victimas\%20del\%2 Odesplazamiento-Andrea\%20Amaya.pdf

Andrade Salazar, J \& et al. (2011). Relación entre indicadores de problemas de adaptación social y un posible trastorno afectivo bipolar (TAB), en mujeres cabeza de hogar en situación de desplazamiento forzado en la ciudad de Armenia en el 2010.www.revistaorbis.org.ve. Descargado de:

http://www.revistaorbis.org.ve/18/art3.pdf

Andrade Salazar, J. (2010) Mujeres, niños y niñas, víctimas mayoritarias del desplazamiento forzado. www.revistaorbis.org.ve. Disponible en: http://www.revistaorbis.org.ve/16/Art2.pdf

Baró Martín, I. (1984). Guerra y Salud Mental. Conferencia pronunciada en San Salvador, en la inauguración de la "I Jornada de Profesionales de la Salud Mental". Publicada en "Estudios Centroamericanos", nº. 429/430, pp. 503-514. Descargado de: http: / / www.dinarte.es/salud-mental/pdfs/MBaro-

Guerra\%20y\%20salud\%20mental.pdf

Ballesteros P; Gaviria L \& Martínez B. (2006). Caracterización del acceso a los servicios de salud en la población infantil desplazada y receptora en asentamientos marginales en seis ciudades de Colombia, 2002-2003. Revista de la Facultad Nacional de Salud Pública, Vol. 24, Núm. 1, pp. 7-17. Universidad de Antioquia, Colombia. Descargado de: http:/ / redalyc.uaemex.mx/pdf/120/12024102.pdf

Bello, M. (2004). Identidad y desplazamiento forzado. Programa Andino de Derechos Humanos - Universidad Andina Simón Bolivar. Revista Aportes Andinos No 8. Desplazamiento forzado y refugio. Disponible en: http://www.uasb.edu.ec/padh/centro/pdfs8/martha\%20bello.pdf

Calvo Ocampo, F. (2009). Mujeres desplazadas en Colombia. Las voces ocultas del conflicto armado. http://www.c3fes.net/docs/sin_nosotrascolombia.pdf

Cortés Ramírez, D \& Parra Alfonso, G. (2009). La ética del cuidado. Hacia la construcción de nuevas ciudadanias. Psicología desde el Caribe. Descargado de: http://ciruelo.uninorte.edu.co/pdf/psicologia_caribe/23/09\%20LA\%20ETICA\%20 DEL\%20CUIDADO.pdf 
Desviat M, et al. (2000). Salud Mental comunitaria como estrategia o utopía. Psiquiatría Pública; 12 (1). Descargado de: http://www.dinarte.es/saludmental/pdf12_1/v12_1.pdf

Foucault, M. (1977). La voluntad de saber, Ed. Siglo Veintiuno.

Gandulfo, M. (2008). Roles de Género y Desplazamiento Interno en Colombia. Centro Argentino de Estudios Internacionales. Programa América Latina. Descargado de: http://www.caei.com.ar/es/programas/latam/15.pdf

Goleman, D. (2004). La inteligencia emocional. Editorial Vergara, Barcelona.

Gómez Palacio, G. (2009). Identidad y sujeto Formación del vínculo social. Revista Electrónica de Psicología Social «Poiésis" ISSN 1692 0945. N 18. Descargado de: http://www.funlam.edu.co/poiesis/Edicion018/Identidadysujeto.GermanGomez.p df

González, C \& Paniagua, C. (2005). Relación entre salud, salud mental e intervenciones psicosociales. Descargado de:

http:/ / www.udea.edu.co/portal/page/portal/bibliotecaSedesDependencias/unida desAcademicas

Guevara Corral, R. Mujeres desplazadas por el conflicto armado. Situaciones de género en Cali y Popayán, Colombia. Universidad del Valle, Asprodeso (Asociación de Profesionales para el Desarrollo Social). Descargado de: http://www.disasterinfo.net/desplazados/informes/asprodeso/mujeresdesplazadas.htm

Guevara Corral, R. (2003). La nueva colonización urbana: el desplazamiento forzado. Reflexión política. Redalyc No 10. Descargado de:

http://redalyc.uaemex.mx/redalyc/src/inicio/ArtPdfRed.jsp?iCve=11051007

Guevara C, R \& Barney Arango, F. (2009). Desplazamiento forzado en florida, valle del cauca. Mujeres, territorio y cultura. Revista Científica Guillermo de Ockham, Vol. 7, Núm. 1, pp. 57-68. Universidad de San Buenaventura, Sede Cali, Colombia. Descargado de: http:/ / redalyc.uaemex.mx/pdf/ 1053/105312251005.pdf

Hernández Delgado, E. (1999). El desplazamiento forzado y la oferta estatal para la atención de la población desplazada por la violencia en Colombia. Universidad Autónoma de Bucaramanga Colombia. Descargado de:

http://redalyc.uaemex.mx/redalyc/pdf/110/11010110.pdf

Ibáñez, M \& Velásquez, A. (2008). El impacto del desplazamiento forzoso en Colombia: condiciones socioeconómicas de la población desplazada, vinculación a los mercados laborales y políticas públicas. División de Desarrollo Social Santiago de Chile. CEPAL Serie Politicas sociales No 145. Disponible en:

http: / / www.eclac.org/publicaciones / xml/2/35022/sps 145-DesplazamientoColombia.pdf

Maturana, Humberto. (1997). La democracia es una obra de arte. Bogotá: Cooperativa Editorial Magisterio. 
Médicos Sin Fronteras. (2010). Tres veces víctimas. Víctimas de la violencia, el silencio y el abandono. Conflicto armado y salud mental en el departamento de Caquetá, Colombia. Disponible en:

http:/ /www.psiquiatria.org.co/BancoMedios/Documentos\%20PDF/lesmesnoticia3 victimas.pdf

Mejía Londoño, A \& córdoba Casas, Y. (2005). Mujer desplazada cabeza de familia informe final. Desplazamiento forzado en Colombia. Universidad nacional de Colombia. Sede Medellin. Facultad de arquitectura. Escuela del hábitat CEHAP. Disponible en: http: / / agora.unalmed.edu.co/desplazados / canal3/proyectos / 2005 01/grupo/MUJER\%20DESPLAZADA\%20CABEZA\%20DE\%20FAMILIA.pdf

Pastrana Arango, A \& et al. (2000). Decreto numero 2569 de 2000. Bogotá, D. C. descargado de: http://www.proyectierras.org/opencms/export/sites/default/public/comunicacio nes/portlets/Documentos/Decretos/DECRETO_2569_DE_2000.pdf

Pécaut, D. (2001). Guerra contra la sociedad. Bogota: Editorial Planeta Colombiana.

Pécaut, D. (2003). Violencia y Politica en Colombia: Elementos de reflexión. Medellin: Hombre Nuevo Editores.

Pécaut, D. (2003). Violencia y Politica en Colombia: Elementos de reflexión. Medellin: Hombre Nuevo Editores.

Profamilia. (2010). Encuesta Nacional de Demografia y Salud. Capítulo 3

Características Generales de los Hogares y de la Población. Disponible en: http://encuestaprofamilia.com/images/stories/PDF-capitulos/Capitulo-3.pdf

Riaño Barrera, E. (2006). De "emprendedor" a "pobrecito". Reestructuración del rol comunitario de familias campesinas desplazadas asentadas en los altos de Cazuca Bogotá. Umbral Científico ISSN 1692-3375. Disponible en:

http://redalyc.uaemex.mx/redalyc/src/inicio/ArtPdfRed.jsp?iCve=30400914.

Serna Ramírez, A. Algunas manifestaciones de la cultura tradicional y popular de una comunidad en situación de desplazamiento: el caso de cercana ilusión. Antípoda, Revista de Antropología y Arqueología, julio-diciembre número 005. Universidad de los Andes, Bogotá, Colombia. Descargado de:

http://redalyc.uaemex.mx/src/inicio/ArtPdfRed.jsp?iCve=81400510

SIPOD \& Acción Social. (2010). Comportamiento del Quindío como receptor y expulsor de población por municipio, año, hogares y personas según el año de valoración. Con corte al 31 de Diciembre 2010. Disponible en:

http://es.scribd.com/doc/50604472/Estadisticas-DF-con-corte-a-diciembre-2010

Lozano A. M \& Gómez G, M. (2004). Aspectos psicológicos, sociales y jurídicos del desplazamiento forzoso en Colombia. Acta colombiana de psicología, numero 012. Universidad católica de Colombia. Bogotá, Colombia. Descargado de: http://regweb.ucatolica.edu.co/publicaciones/psicologia/ACTA/n12/articulosrevi sta/art\%208\%20acta\%2012.pdf 
CODHES (2010). Número de Personas Desplazadas por Municipio de Llegada 1999 - 2010 Fuente: CODHES- SISDHES Fecha de corte: 31 diciembre de 2010 http:/ / www.codhes.org/index.php?option=com_docman\&task=cat_view\&gid=39\&I temid $=51$

Vargas, C \& et al. (1995). Intervención en casos de abuso sexual en estado de crisis. Santafé de Bogotá: Presencia.

Viveros Chavarría, E. (2010). Sentidos de familia y desarrollo en el contexto del desplazamiento de los barrios "Moravia" y "Altos de la Virgen" de Medellín. Una perspectiva crítica Revista Virtual Universidad Católica del Norte, núm. 30, mayoseptiembre, 2010, pp. 1- 23 Fundación Universitaria Católica del Norte Colombia. Descargado de: http:/ / redalyc.uaemex.mx/redalyc/pdf/1942/194214476011.pdf 
